\title{
Sorting criteria. Methods for on-line/at-line sorting of entire male carcasses with emphasis on the Danish method based on skatole content
}

\author{
J Rud Andersen*
}

Address: Danish Meat Research Institute, Denmark

* Corresponding author

from Prevention of Boar Taint in Pig Production: The 19th Symposium of the Nordic Committee for Veterinary Scientific Cooperation Gardermoen, Norway. 2I-22 November 2005

Published: 7 August 2006

Acta Veterinaria Scandinavica 2006, 48(SuppI I):SI4 doi:I0.II86/I75I-0I47-48-SI-SI4

(c) 2006 Andersen.; licensee BioMed Central Ltd.

\section{Introduction}

It has during a long time been a well-known fact that uncastrated male pigs grow faster on less feed compared to castrated ones. In addition, the former yield a higher content of lean meat in the carcass, and they are generally healthier. Consequently, the economic incentive in avoiding castration is strong.

Recently, however, animal welfare aspects have become more dominant than economy, and in certain states legislation is being prepared banning castration altogether, or only allowing castration when the piglets are fully anaesthetized. Together with another fact, i.e. the fact that meat from some uncastrated male pigs exhibits an unpleasant odour when heated, these developments have emphasized the need for being able to sort out the tainted carcasses.

The major compounds responsible for the male odour are known to be the two metabolites of tryptophan, skatole (3-methylindole) $[1,2]$ and indole [3], and the pheromone androstenone (5- $\alpha$-androst-16-en-3-one) [4]. Amongst the three, indole is known to be the less offensive to the human nose. The smell of skatole is described as faecal while the smell of androstenone is described as urinous. Only a fraction of the consumers are capable of smelling androstenone, as this ability is hereditary [5].

Till now, to the knowledge of this author, the only real time, full scale sorting "experiment" conducted under industrial conditions, i.e. directly in an abattoir environment, was carried out in Denmark. A wide variety of minor experiments, all based on laboratory methods for detecting the compounds of interest, have indeed taken place, but none of these come close to being on- or at-line methods. Consequently, a major part of the following discussion is concerned with Danish experience.

\section{Discussion}

Before getting too involved in analytical procedures for detecting "boar taint" compounds, it appears reasonable to look into different sorting scenarios and their consequences. These will be focussed on the two compounds of most significance, skatole and androstenone, as very little has been disclosed on the levels of indole in uncastrated, male carcasses.

Three scenarios come to mind:

Sorting based on skatole

$>$ Sorting based on androstenone

Sorting based on both skatole and androstenone.

With regard to the first, Danish experience has suggested that a range of sorting limits for "skatole equivalents" may be used, and that "skatole equivalents" have a correlation to a trained sensoric panel of 0.76 thus explaining $58 \%$ of the variation (see note 1) [3]. If $0.20 \mathrm{ppm}$ of "skatole equivalents" in backfat is used as a sorting criterion, the occurrence of falsely accepted carcasses, i.e. carcasses that 
possess a deviating smell although accepted from an analytical point of view, is $0.8 \%$ as determined by the sensoric panel. $6.0 \%$ of the carcasses would be rejected.

If, on the other hand, a somewhat higher level of 0.25 ppm of "skatole equivalents" is utilized, the occurrence of falsely accepted carcasses increases to $1.2 \%$ while only $4.3 \%$ is rejected.

Androstenone content by itself explains 24\% of the variation [3], and sorting based on androstenone with a sorting limit of $0.50 \mathrm{ppm}$ in the backfat as proposed by Desmoulin et al. [6] yields the following result on Danish uncastrated male pigs: $1.2 \%$ of the carcasses are falsely accepted, which incidentally is identical to the situation with a 0.25 ppm "skatole equivalents" limit; however, the number of rejected carcasses increases to $48 \%$ !!

By combining the analytical results of "skatole equivalents" and androstenone $66 \%$ of the total variation can be explained. The best combination of the two sets of results will falsely accept $0.4 \%$ of the carcasses and reject $8.7 \%$. Interestingly, in a consumer study carried out on the Danish population of uncastrated male pigs androstenone contents did not appear to have a negative impact on the odour/flavour of the meat at "skatole equivalents" below $0.15 \mathrm{ppm}$. For higher "skatole equivalents" levels the importance of androstenone became noticeable [7]. This means that "skatole equivalents" is a more effective sorting parameter compared to androstenone. However, an effect of both "skatole equivalents" and androstenone on the consumer evaluation appears at extreme values of androstenone (i.e. > 1,5 ppm) as the content of "skatole equivalents" approaches $0.25 \mathrm{ppm}$. Such a situation appears in less than $1 \%$ of the Danish population of entire males [7].

The Danish at-line system for analysis of "skatole equivalents" in backfat from male pigs is based on colourimetry [8]. In the very beginning it started as a laboratory method, which through a series of steps was finally developed into a fully automated analysis robot for use in abattoirs.

In the industrial version the robot has a nominal capacity of 200 samples per hour, of which 20 are used for quality control samples in the form of both liquid standard solutions and fat samples with known contents of analyte. The robot is very accurate and precise, and has built-in alarms for poor performance. Backfat samples are taken from the uncastrated male pigs early on the slaughter line. The time for each individual analysis is approximately 20 minutes, which means that the result is available when the carcass leaves the chilling tunnel at the end of the slaughter line. Carcasses with a content of "skatole equivalents" above the sorting limit $(0.20$ or $0.25 \mathrm{ppm})$ are marked accordingly and kept separate from the other carcasses.

In the 1990's, 17 such robots were installed in Danish abattoirs and a production of uncastrated male pigs took place. In 1993 the number of analyses carried out peaked at around 100,000 per week, but since then the production has almost stopped due to negative reactions to the meat from parts of the international market. These reactions, as far as could be ascertained, arose mostly from the mere fact that the meat originated from male pigs; not because of the presence of any off odours or flavours.

Having stated previously that no other on- or at-line equipment for identifying tainted carcasses from uncastrated male pigs exists, it appears worthwhile to take a look in the crystal ball to find out what the future has in store with regard to boar taint analysis.

Before doing this, however, it must again be mentioned that several successful attempts were made in the last century to develop new analytical methods for either skatole or androstenone contents in uncastrated male pigs, and in a few cases even methods enabling simultaneous determination of both compounds were published and being utilized [9]. They were almost exclusively based on extensive sample pretreatments before detection by means of colourimetry [10], RIA/ELISA [11,12] or chromatographic techniques [13], and although some effort was invested in bringing them from the laboratory to the slaughter line they were never fully implemented.

A number of recent and current attempts are concentrated on technologies that are not dependent on complicated, labour intensive and tedious pretreatment of the fat samples before the actual analysis. (To the mind of the present author this is the only viable way forward.)

The rationale behind such a strategy is obvious: analysis time is minimized, and simultaneous determination of the compounds of interest, - or indeed the direct determination of "odour" or "taint" intensity without quantitation of the compounds responsible for it, - are in principle within the realm of reality.

Different kinds of spectroscopies, such as infrared (IR), near infrared (NIR) or photo acoustic spectroscopy (PAS), have been proposed and/or attempted; either directly on a fat sample, or on the vapour (head space) above a heated fat sample.

Further, promising results were obtained with a number of different "noses", i.e. detectors or arrays of detectors sensitive to molecules in the gas phase above a heated fat sample or originating from the pyrolysis of a fat sample. 
(Unpublished results presented at the "Workshop on Boar detection Methods" Ås, Norway, 21-22 October 2004).

In conclusion, however, although the area has gained enormously in interest during the latest years and many results were achieved with modern and sophisticated technologies, the real break-through is still missing. In other words the only example of a procedure able to discriminate between tainted and untainted carcasses from uncastrated male pigs in an abattoir environment is the somewhat belated Danish one.

\section{Note}

The colourimetric method used previously in Denmark was not designed for determination of only skatole, but was optimized for detection of tainted carcasses using a trained sensoric panel as criterion. It was shown that indole contributes to the odour/flavour of meat from uncastrated carcasses, and the method measures both skatole and indole in the best combination according to the sensoric panel. The results from the method are referred to as "skatole equivalents"; skatole was used as an adequate compound for calibration of the method.

\section{References}

I. Vold E: Report No. 238, Institute of Animal Genetics and Breeding, NLH Volebekk, Norway; 1970.

2. Walstra P, Maarse H: IVO Rapport C-I47 and Rapport No. 2, Researchgroep Vlees and Vleeswaren TNO, Zeist, The Netherlands; 1970.

3. Bejerholm AC, Barton-Gade P: The relationship between skatole/androstenone content and odour/flavour of meat from entire male pigs. In Measurement and Prevention of Boar Taint in Entire Male Pigs Edited by: Bonneau M. INRA Editions, Paris; 1993:75-80.

4. Patterson RLS: 5- $\alpha$-androst-16-en-3-one: compound responsible for boar taint in boar fat. J Sci Food Agric 1968, 19:31-37.

5. Wysocki CJ, Beauchamp GK: Ability to smell androstenone is genetically determined. Proc Natl Acad Sci USA 1984, $8 \mathrm{I}: 4899-4902$.

6. Desmoulin B, Bonneau M, Frouin A, Bidard JP: Consumer testing of pork and processed meat from boars; the influence of fat androstenone level. Livest Prod Sci 1982, 9:707-7I 5.

7. Godt J, Kristensen K, Poulsen CS, Juhl HJ, Bech AC: A consumer study of Danish entire male pigs. Fleischwirtschaft 1996, 76:518-520.

8. Mortensen AB, Sørensen SE: Relationship between boar taint and skatole determined with a new analytical method. In Proceedings of the 30th Europ Mtg. Meat Res. Workers; Bristol; 1984:394-396.

9. Hansen-Møller J: Rapid high-performance liquid chromatographic method for simultaneous determination of androstenone, skatole and indole in back fat from pigs. J Chromatogr 1994, 661:219-230.

10. Squires EJ: Studies on the suitability of a colorimetric test for androst-16-ene steroids in the submaxillary gland and fat of pigs as a simple chemical test for boar taint. Can J Anim Sci 1990, 70:1026-1029.

11. Andresen Ø: A Rapid Radioimmunological Evaluation of the Androstenone Content in Boar Fat. Acta Ve Scand 1979, 20:343-350.

12. Claus R, Mahler G, Muenster E: Determination of the boar taint steroid 5- $\alpha$-androst-16-en-3-one in adipose tissue of pigs with a rapid microtiter plate enzyme immunoassay (MTE). Arch Lebensmittelhyg 1998, 39:87-90.

13. Dehnhard M, Claus R, Hillenbrand M, Herzog A: High-Performance Liquid Chromatographic Method for the determination of 3-Methylindole (Skatole) and Indole in Adipose Tissue of Pigs. J Chromatogr 1993, 616:205-209.

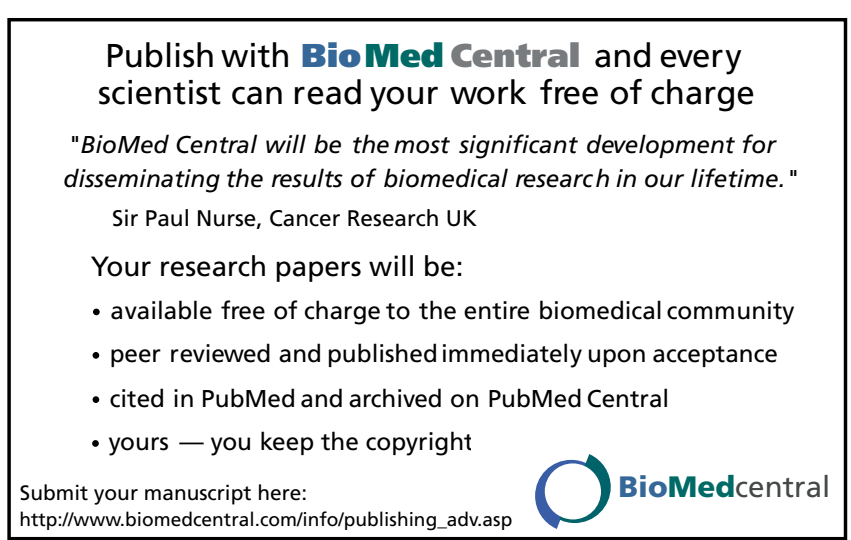

